\title{
Global eigenmodes of free-interface vertical liquid sheet flows
}

\author{
M. Girfoglio, F. De Rosa, G. Coppola \& L. de Luca \\ Dipartimento di Ingegneria Industriale, sez. Aerospaziale, \\ Università di Napoli Federico II, Italy
}

\begin{abstract}
The global dynamics of unsteady free-interface vertical liquid sheet flows is studied, where the dynamics is termed global because it refers to the whole fluid system. The formal development of a proper mathematical model is presented initially, which accounts for pressure disturbances produced by the compliant interface in an air enclosure adjacent to the sheet. The linear spectral analysis (here restricted to sinuous disturbances only) shows that the surface tension is globally stabilizing, the spectrum exhibiting two typical branches related to the two characteristics curves of the governing equations. This basic finding is confirmed by means of both computations of the optimal amplifications (i.e. the greatest amplifications over all initial perturbations) of the relevant system energy and direct numerical simulations of the spatio-temporal evolution of an initial disturbance having the form of an interface Gaussian perturbation.
\end{abstract}

\section{Introduction}

Vertical liquid sheets are often used in industrial processes, classical examples being coating technology and paper making. In most of these applications the control of the global characteristics of the sheet is of great importance for the final quality of the product. The ability in suppressing undesired oscillations or nonuniformity in the steady configuration of the sheet, for example, usually reflects in a good quality of the fluid layer deposited under a moving substrate.

The modeling of the falling liquid sheet and of its interactions with the external ambient gas has been considered in various circumstances in the past, mainly within the context of linear stability analysis (cf. for example [1-4]). In these previous contributions the analysis of the system behaviour is usually conducted 
locally, while few studies have been developed for the global characteristics of the curtain when it strongly interacts with the external ambient gas [5,6]. In this paper, a simple model for the deflection of the liquid sheet is initially derived under the assumptions of thin thickness and small initial perturbations. This model is then closed with the specification of the pressure field outside the sheet, which represents here the reaction of an air enclosure located on one side of the sheet. The equations are then studied theoretically, by employing both modal and non modal analysis, and numerically by a direct numerical simulation of the unsteady (linear) equations governing the evolution of disturbances.

\section{The model}

\subsection{Governing equations}

In order to derive a mathematical model for the unsteady evolution of a freefalling liquid curtain, we will refer to the sketch depicted in Fig. 1. Although in principle various types of interactions with the external gaseous ambient can be considered, hereafter the pressure disturbances produced by the curtain compliant interface within a two-dimensional air enclosure delimited by the curtain itself, rigid walls and a lower basin, will be analyzed. Viscous and splashing effects are neglected. The governing equations are the standard two-dimensional inviscid Euler equations in which gravity is retained. Kinematic and dynamic boundary conditions at the liquid-air interfaces are:

$$
\begin{aligned}
& v^{ \pm}=\frac{\partial y^{ \pm}}{\partial t}+u^{ \pm} \frac{\partial y^{ \pm}}{\partial x} \\
& p^{ \pm}=p_{a}^{ \pm} \mp \sigma \frac{\partial^{2} y^{ \pm}}{\partial x^{2}}\left[1+\left(\frac{\partial y^{ \pm}}{\partial x}\right)^{2}\right]^{-3 / 2}
\end{aligned}
$$

where $\sigma$ is the surface tension coefficient, $y^{-}(x, t)$ and $y^{+}(x, t)$ are the equations of the left and right boundaries of the sheet, respectively, $p_{a}^{-}(x, t)$ and $p_{a}^{+}(x, t)$ are the local values of the external (gas) pressure and the general definition $\varphi^{ \pm}(x, t)=\varphi\left(x, y^{ \pm}, t\right)$ has been employed, $\varphi$ being one of the variables $u, v$ and $p$ (where $u$ and $v$ are the streamwise and lateral velocity components, respectively, and $p$ is pressure).

Let us define the quantities $\widetilde{y}$ and $\bar{y}$ as the thickness and the location of sheet centerline:

$$
\begin{aligned}
& \widetilde{y}(x, t)=y^{+}(x, t)-y^{-}(x, t) \\
& \bar{y}(x, t)=\frac{y^{+}(x, t)+y^{-}(x, t)}{2} .
\end{aligned}
$$

Following Mehring and Sirignano [7], a perturbative approach (in which the thickness of the sheet $\widetilde{y}$ is considered as the small parameter) is adopted, i.e. each 


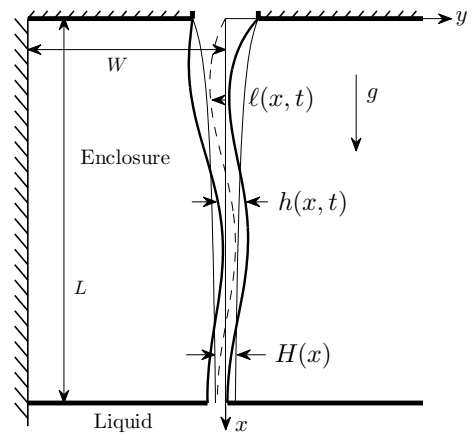

Figure 1: Geometrical configuration under consideration.

dependent variable $\varphi$ is expressed as a power series in terms of $(y-\bar{y})$ :

$$
\varphi=\varphi_{0}(x, t)+\varphi_{1}(x, t)[y-\bar{y}]+\varphi_{2}(x, t)[y-\bar{y}]^{2} \ldots
$$

Such power series expansions are valid for $y \in\left(y^{-}, y^{+}\right)$and hence the inequality $|y-\bar{y}(x, t)| \leq \widetilde{y} / 2$ holds. By adapting the procedure employed in [7], the following set of equations for the lowest order terms in the expansion is derived:

$$
\begin{gathered}
\frac{\partial \widetilde{y}}{\partial t}+\frac{\partial\left(u_{0} \widetilde{y}\right)}{\partial x}=0 \\
\frac{\partial \bar{y}}{\partial t}+u_{0} \frac{\partial \bar{y}}{\partial x}=v_{0} \\
\frac{\partial u_{0}}{\partial t}+u_{0} \frac{\partial u_{0}}{\partial x}=\left[\frac{1}{\rho_{l}\left[\frac{\partial \bar{p}_{a 0}}{\partial x}-\frac{\bar{p}_{a 0}}{\widetilde{y}} \frac{\partial \bar{y}}{\partial x}\right]}\right. \\
+\frac{\sigma}{2 \rho_{l}} \frac{\partial}{\partial x}\left[\left(\frac{f_{+}+f_{-}}{2}\right) \frac{\partial^{2} \widetilde{y}}{\partial x^{2}}\right]+\cdots+\frac{\sigma}{2 \rho_{l}} \frac{\partial}{\partial x}\left[\left(f_{+}-f_{-}\right) \frac{\partial^{2} \bar{y}}{\partial x^{2}}\right] \\
-\frac{\sigma}{\rho_{l} \widetilde{y}} \frac{\partial \bar{y}}{\partial x}\left[\left(f_{+}+f_{-}\right) \frac{\partial^{2} \bar{y}}{\partial x^{2}}+\frac{\left(f_{+}-f_{-}\right)}{2} \frac{\partial^{2} \widetilde{y}}{\partial x^{2}}\right] \\
\frac{\partial v_{0}}{\partial t}+u_{0} \frac{\partial v_{0}}{\partial x}=\left[-\frac{\sigma}{\rho_{l} \widetilde{y}} \widetilde{p}_{a 0}\right]+\frac{\sigma}{\rho_{l} \widetilde{y}}\left[\left(f_{+}+f_{-}\right) \frac{\partial^{2} \bar{y}}{\partial x^{2}}+\frac{\left(f_{+}-f_{-}\right)}{2} \frac{\partial^{2} \widetilde{y}}{\partial x^{2}}\right]
\end{gathered}
$$

where

$$
f_{ \pm}=\left[1+\left(\frac{\partial \bar{y}}{\partial x}\right)^{2} \pm \frac{\partial \bar{y}}{\partial x} \frac{\partial \widetilde{y}}{\partial x}+\frac{1}{4}\left(\frac{\partial \widetilde{y}}{\partial x}\right)^{2}\right]^{-3 / 2}
$$


and $\rho_{l}$ is the liquid density. As usual the following positions are made:

$$
\tilde{p}_{a 0}=p_{a 0}^{+}-p_{a 0}^{-}, \quad \bar{p}_{a 0}=\frac{p_{a 0}^{+}+p_{a 0}^{-}}{2}
$$

in which $p_{a 0}^{ \pm}$are the first order approximation of a power-series expansion in the variable $(y-\bar{y})$ of the (still undefined) ambient gas pressures $p_{a}^{ \pm}$. The boxed terms appearing in equations (7) and (8) are due to the developing nature of the flow under consideration (due to gravity) and were not considered in the original treatment of Mehring and Sirignano [7].

\subsection{Base flow and linearized perturbation equations}

The generic flow variable $\varphi$ will be hereafter decomposed as the sum of a stationary (base) contribution and the perturbation:

$$
\varphi_{0}(x, t)=\Phi(x)+\varphi(x, t) .
$$

Symmetry considerations give the following form for the base flow variables, denoted by capital symbols [8]:

$$
\begin{aligned}
& U=U(x), \quad H=H(x), \\
& V=0, \quad \bar{Y}=0, \quad \widetilde{P}_{a 0}=0, \quad \partial \bar{P}_{a 0} / \partial x=0 \\
& \widetilde{F}=0, \quad \bar{F}=F=\left[1+\frac{1}{4}\left(\frac{\partial H}{\partial x}\right)^{2}\right]^{-3 / 2} .
\end{aligned}
$$

By substituting these position in the nonlinear governing system (5-8) one obtains the following equations for the base flow variables:

$$
\begin{aligned}
& U H=U_{\text {in }} H_{\text {in }}=\text { const. } \\
& U \frac{\partial U}{\partial x}=g+\frac{\sigma}{2 \rho_{l}} \frac{\partial}{\partial x}\left\{F \frac{\partial^{2} H}{\partial x^{2}}\right\}
\end{aligned}
$$

where $U_{\text {in }}$ and $H_{\text {in }}$ are, respectively, the average streamwise velocity and the sheet thickness at the inlet. These equations have been already derived in the context of steady flows and have been extensively studied in [8].

The linearized equations for the perturbation quantities $u, v, \ell$ and $h(l$ referring to the centerline location of the sheet and $h$ to its thickness) are:

$$
\begin{aligned}
& \frac{\partial h}{\partial t}+\frac{\partial}{\partial x}(U h+u H)=0 \\
& \frac{\partial \ell}{\partial t}+U \frac{\partial \ell}{\partial x}=v \\
& \frac{\partial u}{\partial t}+\frac{\partial(U u)}{\partial x}=-\frac{1}{\rho_{l}} \frac{\partial \bar{p}}{\partial x}+\frac{\sigma}{2 \rho_{l}} \frac{\partial^{2}}{\partial x^{2}}\left(F \frac{\partial h}{\partial x}\right) \\
& \frac{\partial v}{\partial t}+U \frac{\partial v}{\partial x}=-\frac{\tilde{p}}{\rho_{l} H}+\frac{2 \sigma}{\rho_{l} H} \frac{\partial}{\partial x}\left(F \frac{\partial \ell}{\partial x}\right)
\end{aligned}
$$


Note that the set of equations (17-20) shows two separated systems for the variables $h, u$ (equations (17) and (19)) and $v, \ell$ (equations (18) and (20)). These two systems will result decoupled if one can show that the values of $\tilde{p}$ and $\bar{p}$ (relative to the ambient gas pressure perturbation) depend respectively on $v, \ell$ and on $u, h$. By following a standard terminology we will employ the term varicose with reference to disturbances involving variations in the thickness $h$ and in the streamwise velocity $u$, and the term sinuous with reference to disturbances involving variations in the centerline position of the sheet $\ell$ and in the transverse velocity $v$.

\subsection{Ambient gas pressure model}

As depicted in Fig. 1, the present model of the external pressure field subjected to perturbation refers to the air enclosure located on one side of the curtain. This type of perturbation, which is induced by a compliant displacement of the sheet interface, was already analyzed by Schmid and Henningson [5], but they did not include surface tension effects. Assuming isentropic transformation of the air within the enclosure and behaviour of perfect gas, yields:

$$
\begin{gathered}
\widetilde{p}=-\frac{\gamma P_{a 0}}{L W-\int_{0}^{L} \frac{H}{2} d x} \int_{0}^{L} \ell d x \\
\bar{p}=\frac{\gamma P_{a 0}}{L W-\int_{0}^{L} \frac{H}{2} d x} \int_{0}^{L} \frac{h}{2} d x
\end{gathered}
$$

where $\gamma$ is the heat specific ratio, $L$ the curtain length and $W$ the transverse dimension of the air enclosure. For varicose disturbances, as shown by equations (19) and (22) the contribution related to the pressure term is null because $\bar{p}$ does not depend on $x$.

\subsection{Non-dimensional equations and slender-sheet approximation}

The equation of motion (15-16) and (17-20) can be conveniently re-written in non-dimensional form by employing the reference quantities:

$$
\begin{gathered}
L_{r}=U_{\text {in }}^{2} / g, \quad \ell_{r}=H_{\text {in }}, \quad u_{r}=U_{\text {in }}, \\
t_{r}=L_{r} / U_{\text {in }}=U_{\text {in }} / g, \\
v_{r}=\ell_{r} / t_{r}=\varepsilon U_{\text {in }}, \quad p_{r}=\rho_{l} g H_{\text {in }}=\varepsilon \rho_{l} U_{\text {in }}^{2}
\end{gathered}
$$

where $\varepsilon=H_{\text {in }} / L_{r}=H_{\text {in }} g / U_{\text {in }}^{2}$ is the slenderness parameter (reciprocal of Froude number). The equations (18) and (20) for sinuous disturbances, for example, reduce to:

$$
\frac{\partial \ell^{*}}{\partial t^{*}}+U^{*} \frac{\partial \ell^{*}}{\partial x^{*}}=v^{*}
$$




$$
\begin{aligned}
\frac{\partial v^{*}}{\partial t^{*}}+U^{*} \frac{\partial v^{*}}{\partial x^{*}}=\frac{1}{\operatorname{We} H^{*}} \frac{\partial}{\partial x^{*}} & \left(F^{*} \frac{\partial \ell^{*}}{\partial x^{*}}\right)- \\
& \frac{\gamma}{H^{*}} \frac{P_{a 0}^{*}}{L^{*} W^{*}-\varepsilon \int_{0}^{L^{*}} \frac{H^{*}}{2} d x^{*}} \int_{0}^{L^{*}} \ell^{*} d x^{*}
\end{aligned}
$$

where $\mathrm{We}=\rho_{l} U_{\text {in }}^{2} H_{\text {in }} / 2 \sigma$ is the Weber number, and $F^{*}$ is:

$$
F^{*}=\left[1+\frac{1}{4} \varepsilon^{2}\left(\frac{\partial H^{*}}{\partial x^{*}}\right)^{2}\right]^{-3 / 2},
$$

stars denoting nondimensional quantities. By adopting a power series expansion in the small parameter $\varepsilon^{2}$, for the base flow variables, and an expansion in $\varepsilon$ for the perturbation quantities,

$$
\begin{aligned}
& U^{*}=U_{0}^{*}+\varepsilon^{2} U_{1}^{*}+\varepsilon^{4} U_{2}^{*}+\ldots \quad v^{*}=v_{0}^{*}+\varepsilon v_{1}^{*}+\varepsilon^{2} v_{2}^{*}+\ldots \\
& H^{*}=H_{0}^{*}+\varepsilon^{2} H_{1}^{*}+\varepsilon^{4} H_{2}^{*}+\ldots \quad \ell^{*}=\ell_{0}^{*}+\varepsilon \ell_{1}^{*}+\varepsilon^{2} \ell_{2}^{*}+\ldots
\end{aligned}
$$

the lowest order approximation for the base flow gives the non-dimensional version of the classic free-fall Torricelli's solution

$$
U_{0}^{*}=\sqrt{1+2 x^{*}}, \quad H_{0}^{*}=\frac{1}{\sqrt{1+2 x^{*}}} .
$$

The sinuous perturbation equations, at zeroth order, are:

$$
\begin{gathered}
\frac{\partial \ell_{0}^{*}}{\partial t^{*}}+U_{0}^{*} \frac{\partial \ell_{0}^{*}}{\partial x^{*}}=v_{0}^{*} \\
\frac{\partial v_{0}^{*}}{\partial t^{*}}+U_{0}^{*} \frac{\partial v_{0}^{*}}{\partial x^{*}}=\frac{1}{\operatorname{We} H_{0}^{*}} \frac{\partial^{2} \ell_{0}^{*}}{\partial x^{* 2}}-\frac{k}{H_{0}^{*}} \int_{0}^{L^{*}} \ell_{0}^{*} d x^{*}
\end{gathered}
$$

where $k=\gamma P_{0}^{*} / L^{*} W^{*}$.

\section{Analysis of sinuous disturbances}

In the present paper we will restrict our study to sinuous disturbances only, that will be analyzed both by determining their global eigenfunctions and by carrying out direct numerical simulations of the governing equations.

\subsection{Linear global eigenfunctions}

Global temporal modes are introduced by the following positions: $\ell_{0}^{*}\left(x^{*}, t^{*}\right)=$ $L_{0}^{*}\left(x^{*}\right) e^{i \omega t^{*}}$ and $v_{0}^{*}\left(x^{*}, t^{*}\right)=V_{0}^{*}\left(x^{*}\right) e^{i \omega t^{*}}$. By substituting them in the governing 


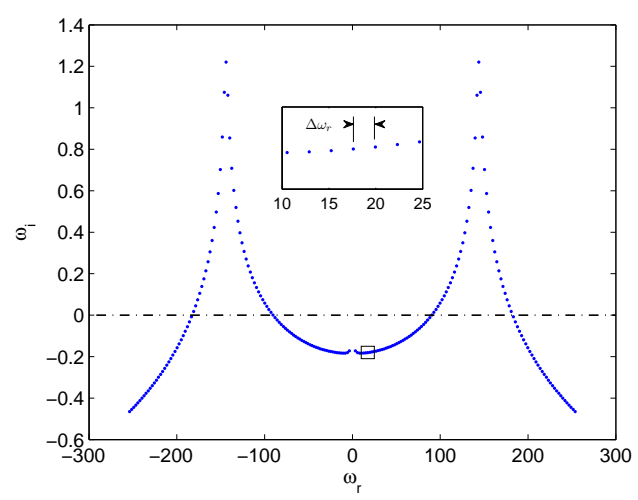

Figure 2: Spectrum for We $=\infty$ and $L^{*}=6.25$.

equations (30)-(31) we obtain

$$
\left[\begin{array}{cc}
I & -U_{0}^{*} D \\
-U_{0}^{*} D & \frac{1}{\mathrm{We} H_{0}^{*}} D^{2}-\frac{k}{H_{0}^{*}} \int_{0}^{L^{*}} d x^{*}
\end{array}\right]\left(\begin{array}{c}
L_{0}^{*} \\
V_{0}^{*}
\end{array}\right)=\mathrm{i} \omega\left(\begin{array}{c}
L_{0}^{*} \\
V_{0}^{*}
\end{array}\right)
$$

where $D, D^{2}$ and $I$ are first derivative, second derivative and identity operators, respectively. The boundary conditions are $L_{0}^{*}(0)=V_{0}^{*}(0)=0$. A Chebychev spectral collocation method has been used in order to solve the eigenvalues problem.

As usual in this kind of problems, once defined the meaningful energy of the system, it is convenient to find the optimal amplification of the energy, i.e. the greatest amplifications for all the possible initial conditions (the so-called growth function $G(t)$ [9]). For sinuous disturbances, the energy is defined as:

$$
E(t)=\int_{0}^{L^{*}} H_{0}^{*} v_{0}^{* 2} d x^{*}+\frac{1}{\mathrm{We}} \int_{0}^{L^{*}}\left(\frac{\partial \ell_{0}^{*}}{\partial x^{*}}\right)^{2} d x^{*}
$$

that takes into account both kinetic and surface tension contributions.

Two typical spectra obtained in the cases of absence of surface tension, We $=$ $\infty$, and We $=2$, both for $L^{*}=6.25$, are depicted in Figs. 2 and 3, respectively.

Note that the former case corresponds to the situation analyzed previously by Schmid and Henningson [5]. In the presence of surface tension the spectrum preserves the symmetry properties, but the unstable modes of high frequency $\omega_{r}$ disappear, due to the stabilizing effects of the surface tension. Moreover, the presence of two distinct symmetrical branches is also evident, which is justified on the grounds of the hyperbolic character of the governing equations system. Neglecting the external pressure contribution, this last can be recast in a single 


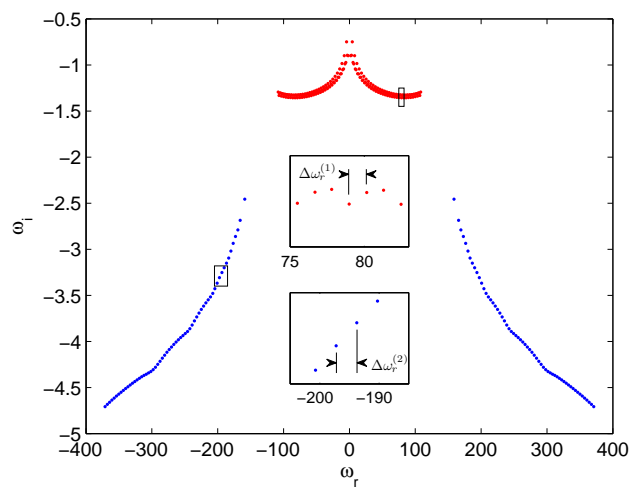

Figure 3: Spectrum for $\mathrm{We}=2$ and $L^{*}=6.25$; the red branch refers to the slower characteristic, the blue branch to the faster one.

equation in terms of $\ell_{0}^{*}$

$$
U_{0}^{*}\left(U_{0}^{*}-\frac{1}{\mathrm{We}}\right) \frac{\partial^{2} \ell_{0}^{*}}{\partial x^{* 2}}+2 U_{0}^{*} \frac{\partial^{2} \ell_{0}^{*}}{\partial x^{*} \partial t^{*}}+\frac{\partial^{2} \ell_{0}^{*}}{\partial t^{* 2}}+\frac{\partial \ell_{0}^{*}}{\partial x^{*}}=0
$$

Equation (34) is a $2^{\text {nd }}$ order hyperbolic partial differential equation having two real and distinct characteristics given by (as an useful reference, see also [4]):

$$
\frac{d x^{*}}{d t^{*}}=U_{0}^{*} \pm \sqrt{\frac{1}{\mathrm{We} U_{0}^{*}}}
$$

Therefore, the two branches of the spectrum correspond to the two characteristics along which disturbances propagate; in order to support further this observation, the fall times (i.e. the times needed in order the sheet perturbation crosses the entire domain) related to the two characteristics,

$$
T_{\text {fall }}^{(1,2)}=\int_{0}^{L^{*}} \frac{d x^{*}}{U_{0}^{*} \pm \sqrt{\frac{1}{\mathrm{We} U_{0}^{*}}}}
$$

have been evaluated $\left(T_{\text {fall }}^{(1)}=1.80, T_{\text {fall }}^{(2)}=5.38\right)$ and related to the almost constant spacing between two subsequent eigenvalues belonging to the same branch measured along the $\omega_{r}$ axis $\left(\Delta \omega_{r}^{(1)}\right.$ and $\left.\Delta \omega_{r}^{(2)}\right)$. It is found that this spacing agrees closely to free-fall frequency $f_{\text {fall }}^{(1,2)}=2 \pi / T_{\text {fall }}^{(1,2)}$. This behaviour was expected, because of some general results on the relation between the global spectrum morphology and oscillations timescales in linear evolutionary models [10]

Fig. 4(a) and (b) show that optimal global amplifications of energy calculated by considering separately eigenfunctions related to two branches of the spectrum exhibit an energy transient growth characterized by time-periodic oscillations with 

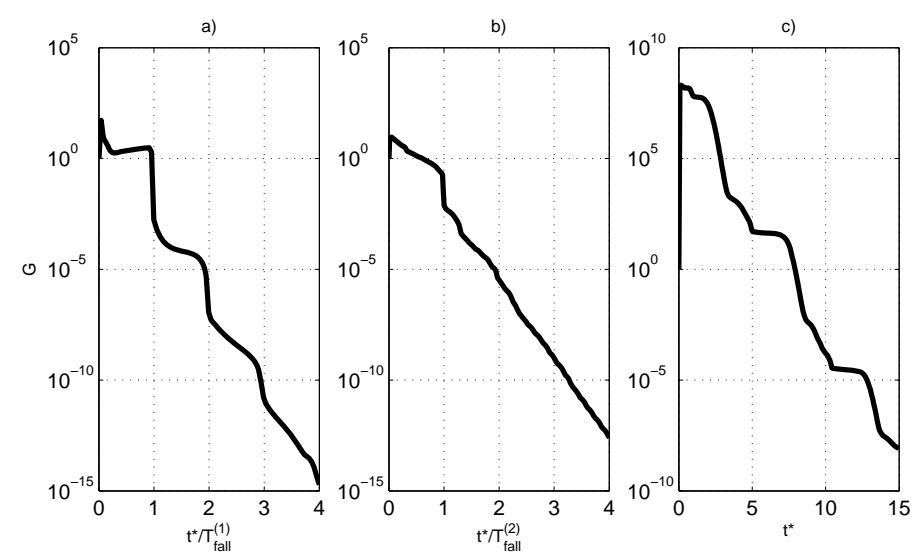

Figure 4: Optimal energy amplifications for $\mathrm{We}=2$ and $L^{*}=6.25$; a) spectrum red branch only; b) spectrum blue branch only; c) overall.

very low peak values. The total gain curve is plotted in Fig. 4(c) where one can observe a very strong transient amplification because of coupling effects between the two branches of eigenvalues.

\subsection{Direct numerical simulation}

In order to validate in another way the results shown in the previous section, direct numerical simulations of the system (30-31), in the absence of the pressure term, have been carried out by using a $4^{\text {th }}$ order finite difference scheme for the discretization of spatial differential operators. For the temporal integration a semiimplicit theta-method scheme has been employed. The spatio-temporal evolution of the initial disturbance constituted by a Gaussian peak for $\ell_{0}^{*}$, located near the origin, is reported in Fig. 5. Note that the initial packet splits while travelling down the sheet according to the different speeds of the two characteristics along with disturbances propagate.

\section{Conclusions}

The unsteady global dynamics behavior of a free-interface vertical liquid sheet flow has been studied, where such a behavior has been defined global because it refers to the entire fluid system as a whole. The first part of the paper dealt with the formal development of a proper mathematical model, which accounts of a particular kind of disturbances in the external gaseous ambient, constituted by an enclosure limited by solid walls, a bottom liquid basin and one of the two sheet interfaces.

A major finding is that the fluid system is stabilized by the surface tension. Moreover, since the unsteady dynamics can be described by means of a typical 


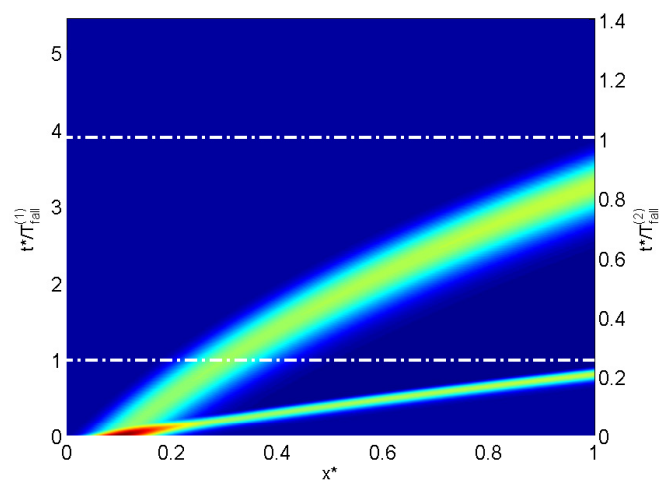

Figure 5: Spatio-temporal evolution of the initial disturbance for $\mathrm{We}=2$ and $L^{*}=1$.

$2^{\text {nd }}$ order partial differential equation of hyperbolic type, showing two distinct characteristic curves, the eigenvalues spectrum is typically characterized by two branches, each of them corresponding to disturbances eigenfunctions propagating along the two characteristic curves.

Another very interesting result is that both spectrum branches highlight a practically constant spacing among the values of their real parts, such a spacing being intimately connected to the fall times of the sheet perturbations over the height of the gas ambient enclosure. This result, which is typical of other convection-dominated dynamics systems, is confirmed under other two viewpoints.

A first comparison basis was offered by direct numerical simulations of the governing equations, that showed, among other features, how the initial disturbance imposed to the flow field under the form an interface Gaussian perturbation, evolved in the spatio-temporal framework by splitting itself into two wave-packets travelling at the expected speeds. In addition, a parallel investigation has been carried out in order to compute time trends of optimal amplifications of the significant system energy (accounting of both kinetic and surface tension contributions), where the term optimal refer to the greatest energy amplification over all initial perturbations. Both comparisons showed results in very close agreement with the spectral analysis performed in the initial part of the paper.

\section{References}

[1] S.P. Lin, Z.W. Creighton and B.J. Creighton, "Absolute and convective instability of a liquid sheet," J. Fluid Mech. 220, 673 (1990).

[2] L. de Luca and M. Costa, "Instability of a spatially developing liquid sheet," J. Fluid Mech. 331, 127 (1997). 
[3] L. de Luca, "Experimental investigation of the global instability of plane sheet flows," J. Fluid Mech. 399, 355 (1999).

[4] S. J. Weinstein, A. Clarke, A. G. Moon and E. A. Simister, "Time-dependent equations governing the shape of a two-dimensional liquid curtain, Part 1: Theory," Phys. Fluids. 9, 3625 (1997).

[5] P. J. Schmid and D. S. Henningson, "On the stability of a falling liquid curtain,” J. Fluid Mech. 463, 163 (2002).

[6] H. Mori, T. Nagamine, R. Ito, Y. Sato, "Mechanism of self-excited vibration of a falling water sheet," Nihon Kikai Gakkai Ronbunshu, C Hen/Transactions of the Japan Society of Mechanical Engineers, Part C 78 (792), pp. 2720-2732 (2012).

[7] C. Mehring and W. A. Sirignano, "Nonlinear capillary wave distortion and disintegration of thin planar liquid sheets," J. Fluid Mech. 388, 69 (1999).

[8] G. Coppola, F. De Rosa and L. de Luca, "Surface tension effects on the motion of a free-falling liquid sheet," in press Phys. Fluids (2013).

[9] P. J. Schmid \& D. S. Henningson, "Stability and Transition in Shear Flows," Springer (2001).

[10] G. Coppola and L. de Luca, "On transient growth oscillations in linear models," Phys. Fluids 18, 078104 (2006). 